\title{
MODELOS DE GESTIÓN PEDAGÓGICA, EN ZONAS DE ATENCIÓN PRIORITARIA: ANÁLISIS DE UNA EXPERIENCIA
}

\author{
Virginia Cerdas Montano ${ }^{1}$ \\ Investigadora y académica, CIDE-Universidad Nacional \\ Heredia, Costa Rica \\ Adyeri Marín Calderón ${ }^{2}$ \\ Investigadora y académica, CIDE-Universidad Nacional \\ Heredia, Costa Rica \\ lleana Vargas Jiménez ${ }^{3}$ \\ Decana del CIDE-Universidad Nacional \\ Heredia, Costa Rica
}

Recibido: 4 de octubre, 2007 - Aprobado: 13 de noviembre, 2007

\begin{abstract}
Resumen: La División de Educación para el Trabajo del CIDE, Universidad Nacional implementa un proyecto de investigación, dirigido a administradores educativos. El objetivo del proyecto es elaborar una metodología para el desarrollo de modelos de gestión pedagógica, que respondan a las necesidades de cada centro educativo participante. Los resultados que se comparten son el producto de una serie de análisis de acción-reflexiónacción. La experiencia es desarrollada, en nueve escuelas, ubicadas en zonas de atención prioritaria. Entre los logros sobresalientes se encuentra la elaboración de modelos de gestión pedagógica propios, que buscan la pertinencia del currículo escolar de acuerdo con cada contexto. Los hallazgos que emergieron serán un aporte para la gestión de centros educativos.
\end{abstract}

Palabras clave: Modelos, gestión, currículo, planificación.

Abstract: The "División de Educación para el Trabajo del CIDE, Universidad Nacional", started a research project for the benefit of educational administrators. The aim of the project is to devise a methodology for the implementation of pedagogical management models, useful to confront the necessities of each educational center. The results are the product of a series of action-reflection-action analysis. The project is being now developed in nine schools located in zones of prioritized focus. Among the most outstanding achievements was the development of their own pedagogical management models, pertinent to the school according to each context. It is expected that the findings will work as a contribution for management in educational institutions.

Key words: Models, management, curriculum, planning.

Máster en Gestión Educativa con énfasis en Liderazgo UNA, se desempeña como investigadora y académica de la División de Educación para el Trabajo.vickycerdas@gmail.com

Licenciada en Ingeniería Industrial, UCR, se desempeña como investigadora y académica de la División de Educación para el Trabajo. adyerimarin@hotmail.com

Máster en Administración Educativa Universidad de Nuevo México, se ha desempeñado como directora, investigadora y académica de la División de Educación para el Trabajo. Actualmente es la Decana del Centro de Investigación y Docencia (CIDE). ivargas@una.ac.cr 


\section{Introducción}

La administración educativa, desde sus orígenes, es vista como una disciplina que debe garantizar la eficiencia y la eficacia en la organización del sistema escolar, razón por la cual los directores $^{4}$ son los llamados a convertirse en gestores de los cambios necesarios para mejorar la calidad de los procesos educativos, y que éstos respondan a las necesidades de la sociedad actual.

El proyecto integrado (Docencia, Extensión y Producción académica) Modelos de Gestión Pedagógica (MGP) fue ejecutado por la División de Educación para el Trabajo (DET) de la Universidad Nacional (UNA); su objetivo principal fue elaborar una metodología para el desarrollo de modelos de gestión pedagógica que respondiera a las necesidades de cada centro educativo participante, con el fin de actualizar los perfiles de los profesionales en administración educativa, y pudieran, así, promover los cambios requeridos en estos centros, los cuales están ubicados en zonas de atención prioritaria de San José y Heredia. Además, se coordinó, a lo largo de la ejecución del proyecto (2005 a 2007), con el Programa PROMECUM, del Ministerio de Educación Pública (MEP).

En este artículo se plantea cómo la gestión educativa debe responder a un proceso dinámico, innovador, renovador y participativo. Para que estas características se concreten en los centros educativos, necesariamente, se requieren directores que posean un perfil, que les permita desarrollar sus talentos y ejercer un liderazgo pedagógico, para crear el sentido de sus instituciones. Mintzberg (citado por Jabif, en Braslavky, Acosta y Jabif, 2004) considera que "el líder educativo debe efectuar una integración entre las necesidades personales, las grupales y las institucionales, en función de ciertos objetivos organizadores de las prácticas" (p. 8).

De esta manera, se favorece que las personas involucradas dentro del proyecto alcancen una participación activa en todos los procesos que comprende la gestión, logrando establecer mecanismos de mejoramiento continuo en sus centros educativos. Sin olvidar que las necesidades de las escuelas de zonas de atención prioritaria, en las que surgió el proyecto,

En este documento de utiliza, de manera general, el género gramatical masculino, únicamente con el objeto de hacer más expedita su escritura y clara su lectura. No pretende ningún tipo de discriminación sexual. 
están inmersas en contextos tradicionalmente excluidos, donde existen problemas de deserción, de agresión familiar, de violencia doméstica, entre otros.

Acercarse a comprender estas realidades no fue tarea sencilla, ya que implicó desarrollar el sentido de pertenencia de todos los actores sociales inmersos en el proceso. Es necesario un cambio dentro de la gestión escolar, que comprenda no sólo lo curricular, sino, también, el componente organizativo, que involucre a toda la comunidad educativa, entendiendo a esta última como la integrada por el personal docente y administrativo, el alumnado, los responsables legales y la sociedad civil, con el fin de generar propuestas colectivas y sostenibles.

\section{Antecedentes del proyecto}

El proyecto inició con 29 centros educativos en el año 2005; sin embargo, para el mes de setiembre de 2007 sólo se contaba con 9 de esos centros educativos, los cuales llegaron a la etapa de implementación del Modelo de Gestión Pedagógica (MGP), que se presenta más adelante.

El Consejo Superior de Educación, como ente regulador del sistema educativo, faculta a los administradores de los centros, la libertad para favorecer los cambios que las circunstancias exijan; es, por ello, que la figura del director como líder pedagógico cobra gran importancia para gestar nuevas alternativas que permitan articular el ideario societal con la formación que se ofrece en los salones de clases.

Como lo plantea Franco, en Programa de las Naciones Unidas para el Desarrollo (1999):

No es, pues, causal que la educación sea la inversión social con las más altas tasa de retorno, tanto para la sociedad como para los individuos. Ella permite un aumento progresivo de la disponibilidad de capital humano, prepara a la economía nacional para la incorporación sostenida de progreso técnico en la esfera productiva, y permite mayor integración cultural y participación en la vida pública (p. 86).

Por lo anterior, se planteó que los centros educativos requerían de un cambio que emanara de la propia gestión interna; además, el Plan de acción de la educación para todos 
2003-2015 plantea una serie de habilidades para el relanzamiento de la educación costarricense. Entre las principales estrategias que justifican la necesidad de un cambio urgente, según el duodécimo Informe Estado de La Nación (2005) están:

- $\quad$ La educación primaria de calidad.

- $\quad$ La incorporación de los grupos excluidos.

- $\quad$ El programa nacional de formación de valores.

- $\quad$ El plan de prevención y atención de la violencia en y desde los centros educativos.

- $\quad$ El trabajo conjunto entre el centro educativo y la comunidad.

- La tecnología y la educación.

- $\quad$ La gestión administrativa.

Este cambio se concreta por medio de un modelo de gestión pedagógica, el cual se concibe como una respuesta que cumple con las políticas nacionales y, a la vez, con las necesidades locales, y no como una respuesta descendente, sino más bien ascendente, es decir, dinámica e innovadora que se ajusta a cada centro educativo, porque emana de los intereses y las necesidades de los propios contextos.

En el resumen del undécimo Informe del Estado de la Nación (2004) se establece que: "En la educación primaria las necesidades más apremiantes son las aulas académicas, de preescolar y para asignaturas especiales, así como la insuficiencia en áreas como gimnasios, salas para el personal docente, mallas para la protección de las escuelas, laboratorios de ciencias, bibliotecas y computadoras" (pp. 16-17).

Además, en el mismo resumen se establece que, aunado a los problemas de infraestructura, los factores sociales, económicos y geográficos, la deserción, el rendimiento académico y el acceso a la tecnología son problemas que deben atenderse para mejorar la calidad de la educación costarricense.

Por lo anterior, se plantea la necesidad de que los centros educativos generen, de acuerdo con sus capacidades, desde su interior las respuestas a estos problemas; por lo que se propone la formulación de los Modelos de gestión pedagógica, para solucionarlos. 


\section{¿Qué es el Modelo de Gestión Pedagógica?}

El MGP es un método orientado a estructurar los pasos o fases que se deben seguir para la innovación en los centros educativos. Presenta herramientas clave que permiten a una institución lograr los objetivos propuestos.

A continuación, se detalla, especialmente, la primera fase del diagnóstico, ya que de éste dependen las fases por seguir. Y, aunque todo proceso de cambio inicia con la definición del problema por solucionar, la pertinencia de éste depende de contar con información objetiva y actualizada, por lo que el proceso inicia con un diagnóstico (véase la Figura № 1).

\section{MGP Fase 1. Diagnosticar}

Este diagnóstico constituye la primera fase del MGP y debe incluir la siguiente información:

- Intereses de los clientes. Consiste en definir el cliente o los clientes que hay que satisfacer, así como sus necesidades e intereses. Existen diferentes clientes que un centro educativo debe satisfacer: los estudiantes (sus clientes directos), los responsables legales de éstos, los docentes, el personal administrativo, el Ministerio de Educación Pública y la sociedad costarricense.

- Entorno comunitario. Deben establecerse, claramente, las condiciones actuales de la comunidad en la que está inmerso el centro educativo, las características geográficas, climáticas (si pudieran afectar la actividad del centro educativo), económicas y sociales.

- Identificación del problema. Una vez clarificado el entorno y las necesidades de los clientes, se puede iniciar el planteamiento del problema o necesidad por solucionar; sin embargo, éste no es todavía definitivo hasta que se conozcan las capacidades y las restricciones institucionales.

- Entorno educativo. Éste incluye diversos aspectos: primero, se debe conocer cuáles son las restricciones del centro, ya que todo lo que se diseñe debe tenerlas en cuenta para 
que no sean un obstáculo. Además, si el centro educativo cuenta con objetivos ya definidos debe verificarse su interrelación, coherencia y cumplimiento con la gestión que se lleve a cabo en ese momento.

- $\quad$ Procesos actuales. La revisión y el análisis de las condiciones en que labora el centro educativo, permite disponer de datos válidos y suficientes para iniciar los procesos de mejoramiento.

- Análisis FODA. Este análisis permite conocer la posición interna del Centro, en función de sus debilidades y fortalezas; y de la externa, con sus: oportunidades y amenazas. De esta forma, se pretende que el Centro, obtenga ventaja de sus fortalezas, y cuide y fortifique aquellos aspectos clasificados como débiles. Por otra parte, el conocimiento interno permite hacer frente a las amenazas del entorno y aprovechar las oportunidades. La importancia de este tipo de análisis se acentúa bajo el supuesto de que un conocimiento interno, puede convertir las amenazas en oportunidades.

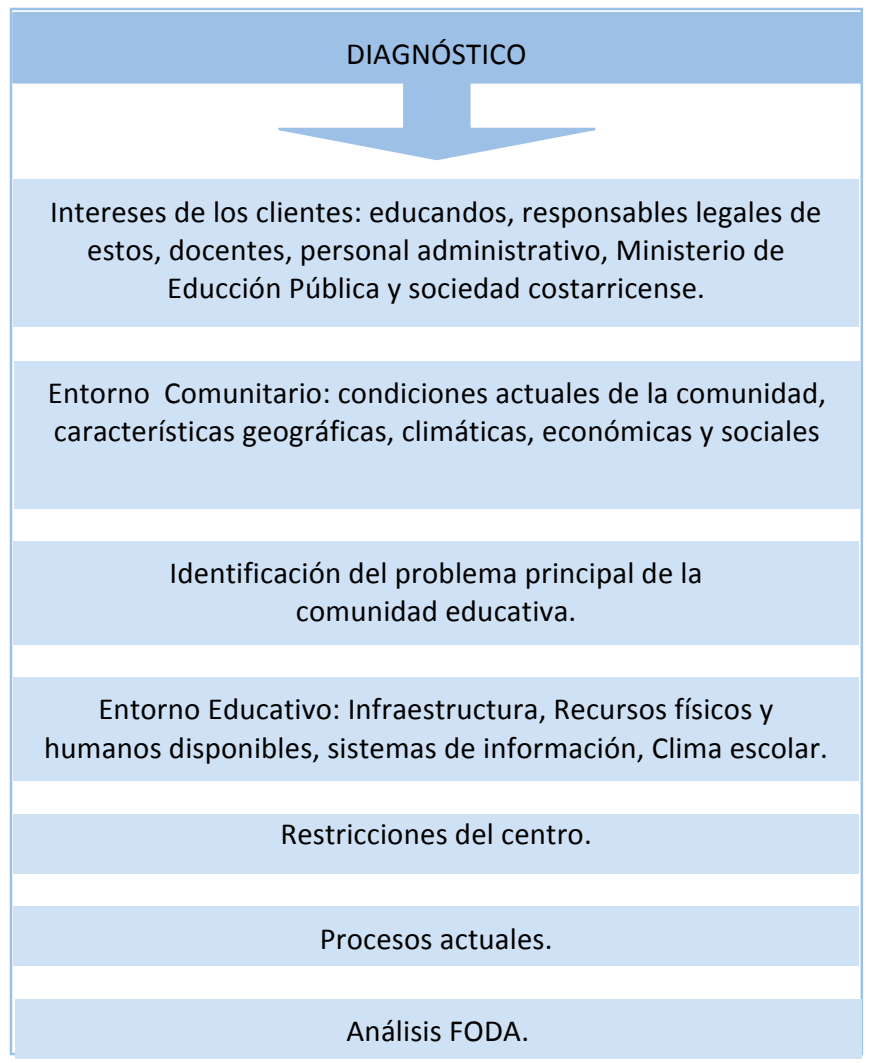

Figura № 1. Diagnóstico del MGP. 


\section{MGP Fase2. Planear}

Una vez finalizado el diagnóstico se verifica, nuevamente, el problema y se ajusta, en caso de ser necesario, para que, con base en éste, se inicie la planificación del modelo que comienza con la verificación de la legislación aplicable, los requerimientos del MEP y el propio diagnóstico.

Esta etapa del MGP tiene como objetivo principal determinar y clarificar hasta dónde quiere llegar el centro educativo; definir, paso a paso, las diferentes etapas o estrategias que deben realizarse para la implementación del rol seleccionado.

El centro educativo debe definir su razón de ser, es decir, su misión, visión y rol institucional, que serán la base para la definición de los objetivos estratégicos y operativos, de lo contrario los planes de acción no tendrán coherencia entre sí o no se integrarán encaminando al centro educativo a un resquebrajamiento. Es necesario establecer las metas de los objetivos, así como los indicadores que son necesarios para verificar su cumplimiento.

Es importante recalcar que estos indicadores son de gestión. El error común es plantear indicadores operativos, y en demasía, pues se debe pensar sólo en los necesarios y claves para la toma de decisiones. Deben plantearse adecuada, clara y oportunamente, reduciendo, así, el riesgo o el oportunismo.

\section{MGP Fase 3. Implementar}

Se pone en marcha todo lo planeado, verificando, a cada paso, su realización, de acuerdo con los indicadores y la información que se va recibiendo al ejecutar cada actividad, con el fin de analizar y verificar el cumplimiento de los objetivos y la posibilidad de logro. En caso contrario, se deben tomar las acciones correctivas.

En esta fase, la comunicación es de vital importancia para reducir la resistencia al cambio y facilitar el proceso de implementación, así como para obtener la realimentación adecuada y oportuna, para llevar a cabo los ajustes durante la marcha. 
El entrenamiento o capacitación proporcionará a los integrantes las destrezas, las habilidades y los conocimientos adecuados para realizar las nuevas actividades; asimismo, permitirá establecer los nuevos métodos de trabajo y la organización del centro, y explicar los controles operativos: cuáles son, cuál es su uso y el por qué de su importancia.

MGP Fases 4 y 5. Verificar y Ajustar

Estas fases están totalmente ligadas entre sí y con la fase 3, ya que no se debe dar tiempo para realizar los ajustes; la verificación contra los parámetros establecidos y las metas propuestas requieren de ajustes prontos y solícitos, con el fin de reducir el riesgo de fracaso del MGP (véase la Figura № 2).

Si se encuentran diferencias entre lo planificado y lo ejecutado, no sólo se deben dar ajustes, sino que, también, es necesario analizar la causa de tal brecha y constituir acciones correctivas que la minimicen o eliminen, así como plantear las acciones preventivas para que la situación no se repita en el futuro. Sin embargo, se establece que, al menos una vez al año, se lleve a cabo una revisión total del modelo por parte de la Dirección del centro educativo, para que se realicen todos los ajustes estratégicos necesarios, y no sólo los operativos.

Finalizado este ciclo, inicia de nuevo, de tal manera que permita al centro educativo generar una cultura de mejoramiento continuo y de adaptación a las necesidades de la sociedad dinámica en la que se encuentra inmerso.

Por tanto, el MGP se integra de las siguientes fases; diagnóstico, planificación, implementación, verificación y ajuste. Éstas corresponden al ciclo de mejoramiento continuo, que se muestra en la siguiente figura; en ella se presenta el modelo reducido por la complejidad de cada una de los pasos.

Debe recordarse que el diagnóstico es una actividad constante, con el fin de tener la información necesaria para prever el cambio o ajustarse a él lo más pronto posible y, así, que el centro educativo sea realmente una organización actualizada. 


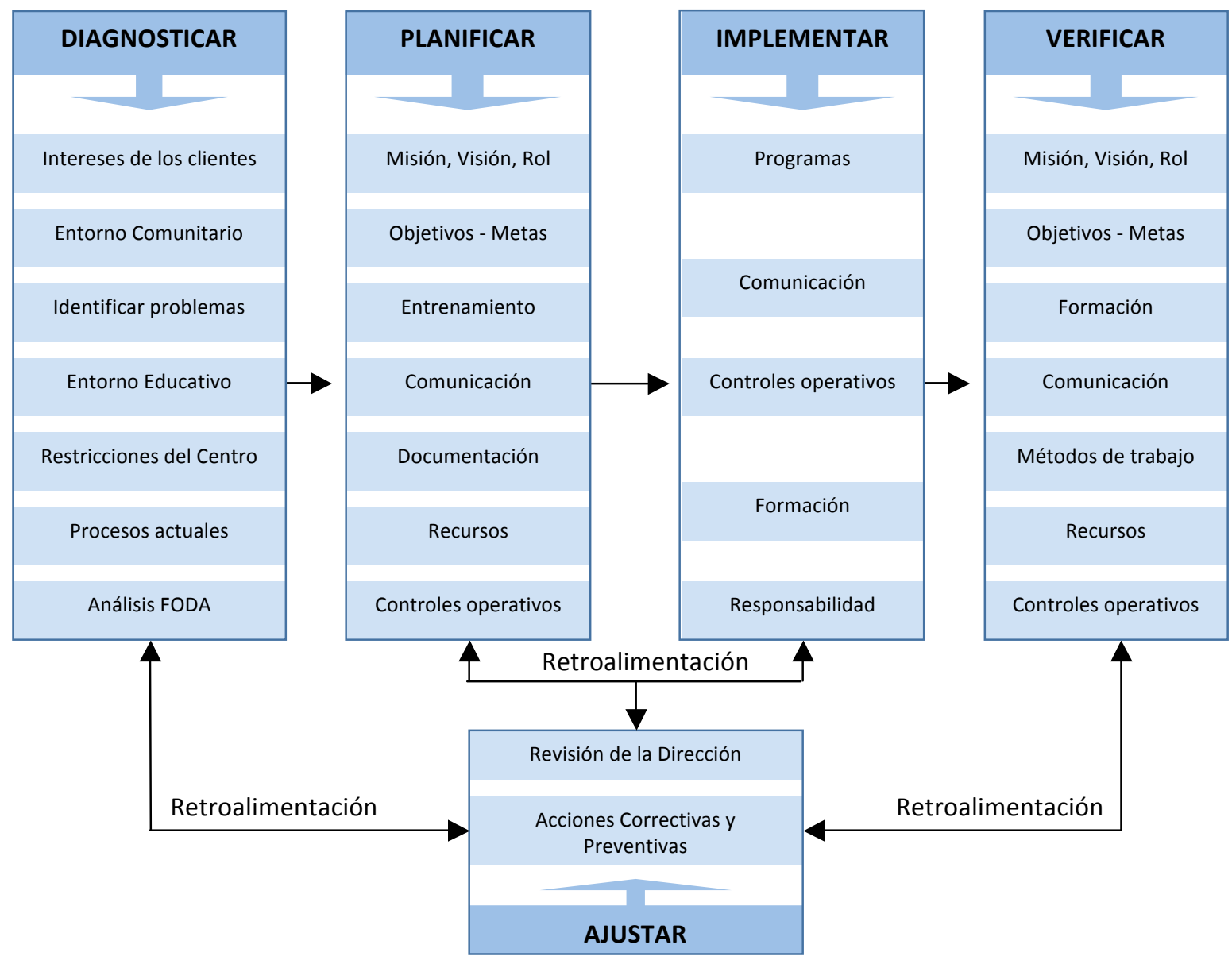

Figura № 2. Modelo de gestión pedagógica reducido.

\section{Realidades de las instituciones educativas participantes}

Las instituciones educativas, a veces, están inmersas en ambientes dinámicos; otras, en entornos problemáticos. Esto último, no sólo es una realidad para Costa Rica, sino a escala mundial. En la mayoría de los casos, las situaciones problemas llevan algunos años de vigencia, sin que se llegue a soluciones que permitan a las organizaciones un funcionamiento más efectivo.

Al respecto, Domínguez y Mesanza (1996), en la conferencia sobre la rendición de cuentas de los programas escolares en perspectiva al siglo XXI, señalan que lograr una educación de alta calidad es una tarea compleja, y que requiere de un consenso social para determinar lo que es esencial para una enseñanza de calidad. Es necesario llevar a cabo un análisis de las organizaciones educativas en varios aspectos, tales como sus principios, los cuales son, frecuentemente, interpretados desde diferentes enfoques curriculares. Entre ellos se podrían 
citar: diversidad, equidad, igualdad, calidad, educación permanente de los ciudadanos, o dicho en otras palabras, oportunidades educativas compatibles con las habilidades y las destrezas del individuo, en relación armoniosa con sus contextos culturales.

Otro de los aspectos por analizar es la urgencia de crear el sentido del currículo en los centros educativos, en todos los niveles de enseñanza; a esto se le une la necesidad de liberar la enseñanza y a los docentes de ahogos administrativos, lo que no les permite ocuparse del eje principal que se debe desarrollar en todo centro de enseñanza: el currículo. Lo anterior evidencia la necesidad de innovar en los centros educativos, para que su oferta sea pertinente a los cambios sociales de los contextos, en los que se encuentran inmersos. Se requiere de organizaciones dinámicas, con un norte claro de su proyecto educativo, integradoras de los elementos que intervienen en el desarrollo del currículo. Según Domínguez y Mesanza (1996), en las organizaciones debe existir una serie de aspectos para facilitar la implementación del currículo; entre ellas están:

- $\quad$ La interrelación de elementos diversos (materiales, personales, de infraestructura

- La instrumentación para lograr una meta o un fin.

- $\quad$ El control constante y su evaluación.

- $\quad$ El dinamismo intra, entre y extra coordinador entre los diferentes elementos.

- $\quad$ El sentido de aplicabilidad y adecuación a la realidad.

- $\quad$ La continuidad en el tiempo.

Lamentablemente, la realidad de nuestros centros educativos muestra una situación muy opuesta a las sugerencias de Domínguez y Mesanza (1996), quienes consideran que al centro educativo, como sistema social, se le han asignado funciones específicas, tales como las de potenciar la formación, adaptación social y facilitar el aprendizaje. Se debe tomar en cuenta que el Centro está conformado por un colectivo de personas (padres, madres, docentes, alumnos, alumnas, personal administrativo) con diferentes intereses, creencias, objetivos, patrones de comportamiento, roles y necesidades que, a veces, dificultan su organización. 
Estas características se reflejan en el estudio realizado por, Gairín (citado por Domínguez y Mesanza 1996), en el que se hace referencia a las características que predominan en los centros educativos, y que deben fortalecerse; entre ellas: la falta de definición de metas, la naturaleza de ellas, la ambigüedad de tecnologías, la falta de preparación técnica, una marcada debilidad del sistema y, por último, la vulnerabilidad de los centros educativos.

Con este panorama sobre la realidad de los centros educativos, se desarrolla la práctica que se desea compartir, con la intención de que pueda tomarse como marco de referencia para otras similares.

\section{Análisis retrospectivo de la experiencia}

Es importante señalar las etapas implementadas durante los tres años de ejecución del proyecto; identificando los aciertos y los desaciertos que resultan de la toma de decisiones en procesos de mejoramiento continuo, la siguiente figura muestra el resumen del proceso desarrollado, el cual se explica después de ella.

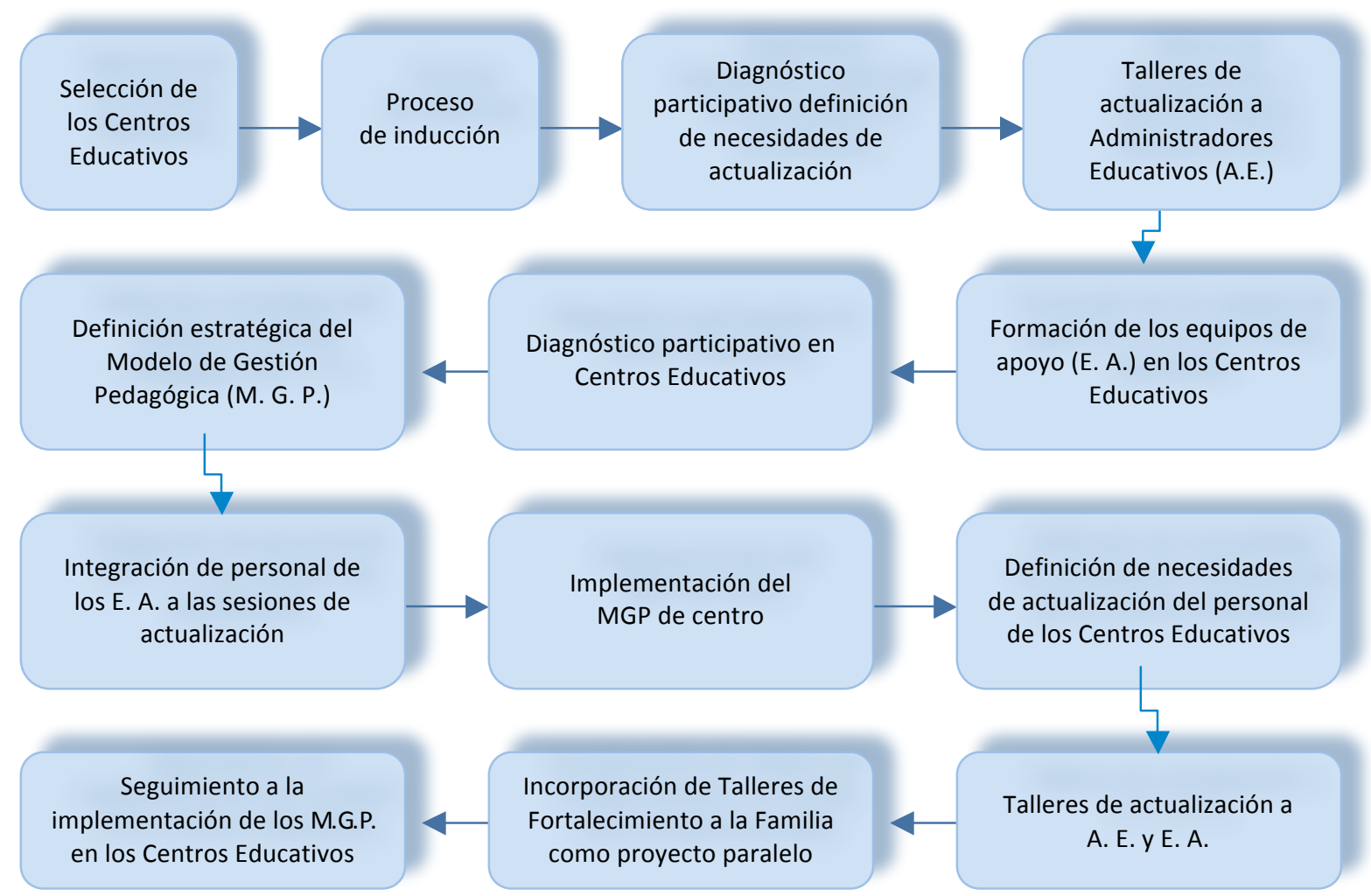

Figura № 3. El proceso del proyecto. 
Pueden observarse, de manera completa, las fases del proyecto: desde la fase inicial (selección de los centros y la inducción de los participantes).hasta la fase final (seguimiento a la implementación de los MGP).

Las tablas que se presentan a continuación muestran los principales aciertos y desaciertos encontrados durante todo el proceso del proyecto. Cabe destacar el apoyo recibido por parte del Viceministro Académico de turno, el M. Sc. Wilfrido Blanco, hecho clave para el inicio y el desarrollo del proyecto en su primer año; otro aspecto importante fue la preocupación de los directores para poder asistir a las sesiones de trabajo en la Universidad Nacional.

Tabla № 1

Fase inicial

\begin{tabular}{|l|l|}
\hline \multicolumn{1}{|c|}{ Aciertos } & \multicolumn{1}{|c|}{ Desaciertos } \\
\hline $\begin{array}{l}\text { Apoyo de las autoridades superiores del Ministerio } \\
\text { de Educación Pública al proyecto. }\end{array}$ & $\begin{array}{l}\text { Ausencia los asesores supervisores de circuitos } \\
\text { escolares y los directores regionales. }\end{array}$ \\
\hline $\begin{array}{l}\text { Obtención de permisos para la participación de los } \\
\text { directores en las sesiones de trabajo. }\end{array}$ & \\
\hline $\begin{array}{l}\text { Anuencia de los directores para participar en las } \\
\text { sesiones de trabajo. }\end{array}$ & \\
\hline
\end{tabular}

Para una adecuada ejecución de este tipo de proyectos es necesaria la participación y el apoyo de los asesores supervisores, ya que el desconocimiento de las necesidades de los centros para ejecutar los cambios requeridos, representa, para algunos de ellos, una limitante.

En la Tabla № 2 se integran los aciertos y los desaciertos del diagnóstico de necesidades de capacitación realizado en la primera sesión de trabajo con los directores, lo que ayudó a formular y planificar 10 sesiones de trabajo, con las siguientes temáticas:

- Inteligencia emocional

- $\quad$ Liderazgo-teorías

- Evaluación institucional

- Modelos gerenciales en administración educativa

- Vitalidad organizacional 
- $\quad$ Trabajo en equipo y

- Comunicación asertiva

Tabla № 2

Diagnóstico de necesidades y talleres

\begin{tabular}{|l|l|}
\hline \multicolumn{1}{|c|}{ Aciertos } & \multicolumn{1}{c|}{ Desaciertos } \\
\hline $\begin{array}{l}\text { Planificación de los talleres a partir de las } \\
\text { necesidades específicas de los participantes. }\end{array}$ & $\begin{array}{l}\text { El tiempo asignado para la realización de los talleres se } \\
\text { torna insuficiente ante el entusiasmo e interés } \\
\text { mostrado. }\end{array}$ \\
\hline $\begin{array}{l}\text { Las temáticas sugeridas por los participantes al ser } \\
\text { paradigmas emergentes en el campo de la } \\
\text { administración educativa, permitieron la } \\
\text { actualización del equipo investigador. }\end{array}$ & $\begin{array}{l}\text { Los recursos asignados al proyecto se tornan } \\
\text { insuficientes ante la demanda de material de apoyo. }\end{array}$ \\
\hline $\begin{array}{l}\text { Se evidenció un interés por parte de los participantes, } \\
\text { mediante las diferentes dinámicas desarrolladas. }\end{array}$ & \\
\hline $\begin{array}{l}\text { Se logró sistematizar el material de apoyo para cada } \\
\text { una de las sesiones, lo que permitió replantearse, } \\
\text { profundizar, analizar y reflexionar sobre las temáticas } \\
\text { definidas. }\end{array}$ & \\
\hline
\end{tabular}

Las temáticas que se establecieron responden a las necesidades sentidas por los participantes desde su experiencia profesional.

La metodología empleada por el equipo investigador se centró en el análisis de situaciones reales, lo que ayudó a generar procesos de reflexión en torno a la práctica profesional.

El proceso diagnóstico de cada centro educativo, permitió un mayor acercamiento de los equipos de apoyo a las necesidades que viven los diferentes actores sociales. La puesta en común sobre las percepciones de los docentes, de acuerdo con el diagnóstico institucional, genera compromiso para la búsqueda de soluciones a los problemas detectados.

Es importante recalcar que se creyó que los directores estaban comunicando y trasladando la experiencia de los talleres al resto del personal, pero al integrar los equipos de apoyo, se evidenció que no fue así. Por tanto se generó un retroceso en el proceso, especialmente en las instituciones en las que los directores fueron trasladados a otros centros y los equipos tuvieron que conocer mejor el proyecto (véase la Tabla № 3). 
Tabla № 3

Formación de equipos de apoyo y diagnóstico de centros educativos

\begin{tabular}{|l|l|}
\hline \multicolumn{1}{|c|}{ Aciertos } & \multicolumn{1}{|c|}{ Desaciertos } \\
\hline $\begin{array}{l}\text { Los equipos de apoyo son conformados por } \\
\text { docentes de los diferentes niveles, equipos } \\
\text { interdisciplinarios y profesores de áreas especiales, } \\
\text { lo que permite una mayor representatividad de las } \\
\text { necesidades vividas en la institución. }\end{array}$ & $\begin{array}{l}\text { La conformación de los equipos de apoyo se establece } \\
\text { en la segunda etapa del proyecto. }\end{array}$ \\
\hline $\begin{array}{l}\text { El diagnóstico permite la obtención de información } \\
\text { acerca de las necesidades, intereses e inquietudes } \\
\text { de los diferentes actores sociales. }\end{array}$ & $\begin{array}{l}\text { Carencia de destrezas (de los equipos de apoyo) en el } \\
\text { manejo de cuadros estadísticos para presentar la } \\
\text { información obtenida. }\end{array}$ \\
\hline $\begin{array}{l}\text { Se cuenta con documentación válida y actualizada lo } \\
\text { que permite el inicio del modelo de gestión } \\
\text { pedagógica. }\end{array}$ & $\begin{array}{l}\text { Los directores no bajaron la información del proceso a } \\
\text { los docentes. }\end{array}$ \\
\hline
\end{tabular}

Los MGP se consideraron una construcción social en este proceso, ya que los participantes fueron los que develaron sus múltiples necesidades, para responder, de manera efectiva y eficiente, ante las demandas del contexto en el que se encuentran inmersos sus centros educativos.

Tabla № 4

Definición estratégica del MGP e integración de los equipos de apoyo a las sesiones de actualización

\begin{tabular}{|l|l|}
\hline \multicolumn{1}{|c|}{ Aciertos } & \multicolumn{1}{c|}{ Desaciertos } \\
\hline $\begin{array}{l}\text { El avance en la planificación de los modelos de } \\
\text { gestión pedagógica se flexibiliza para cada centro } \\
\text { educativo. }\end{array}$ & $\begin{array}{l}\text { El esquema del modelo de gestión pedagógica, se } \\
\text { torna complejo para los participantes. }\end{array}$ \\
\hline $\begin{array}{l}\text { La participación de los equipos de apoyo, en las } \\
\text { sesiones de actualización, permiten brindar espacios } \\
\text { de reflexión y análisis desde diferentes perspectivas. }\end{array}$ & $\begin{array}{l}\text { El tiempo para los espacios de reflexión se reduce por } \\
\text { la cantidad de participantes. }\end{array}$ \\
\hline
\end{tabular}

Durante la fase de implementación, los directores de los centros educativos pusieron en práctica sus saberes conceptuales y los convirtieron en saberes operativos, mediante la transferencia de conocimientos de la teoría narrativa a la acción. La rotación del personal, junto con el hecho de que no se trasladó toda la información del proceso al personal, implicó que se tuviera que reiniciar el proceso en algunos centros (véanse las Tablas № 4 y № 5). 
Tabla No. 5

Implementación de los MGP y definición de necesidades de actualización del personal en cada centro educativo

\begin{tabular}{|l|l|}
\hline \multicolumn{1}{|c|}{ Aciertos } & \multicolumn{1}{c|}{ Desaciertos } \\
\hline $\begin{array}{l}\text { Se realizan visitas por parte del grupo investigador } \\
\text { para apoyar las iniciativas que ha generado el MGP } \\
\text { en cada centro educativo. }\end{array}$ & $\begin{array}{l}\text { La rotación del personal administrativo en las escuelas } \\
\text { participantes, dificultan la buena marcha de la } \\
\text { implementación del modelo. }\end{array}$ \\
\hline $\begin{array}{l}\text { La implementación de algunas acciones generadas } \\
\text { en el MGP, se observan a través de bitácoras, } \\
\text { informes parciales y murales, entre otros. }\end{array}$ & $\begin{array}{l}\text { La cantidad de requerimientos solicitados por el MEP, } \\
\text { es una limitante en la implementación del MGP. }\end{array}$ \\
\hline
\end{tabular}

Para la segunda fase, se integró otro proyecto de investigación que la DET tiene adscrito, el cual se denomina Fortalecimiento de la familia, ya que un elemento importante en la formación de los educandos es el soporte que los responsables legales les brinden. La Tabla № 6 muestra cómo esta incorporación ha permitido brindar a los equipos de apoyo y al resto del personal de la institución estrategias para integrar a los responsables legales en la formación de los educandos.

Tabla No. 6

Seguimiento a la implementación de los MGP e incorporación al Proyecto Fortalecimiento de la familia

\begin{tabular}{|l|l|}
\hline \multicolumn{1}{|c|}{ Aciertos } & \multicolumn{1}{c|}{ Desaciertos } \\
\hline $\begin{array}{l}\text { Contacto permanente con los equipos de apoyo de } \\
\text { cada centro educativo. }\end{array}$ & $\begin{array}{l}\text { El traslado a cada centro educativo participante } \\
\text { implica una inversión de tiempo y gastos por los } \\
\text { miembros del equipo investigador, que no se } \\
\text { contemplan en el presupuesto del proyecto. }\end{array}$ \\
\hline $\begin{array}{l}\text { Incorporación de los equipos de apoyo al proyecto } \\
\begin{array}{l}\text { Educación para el Trabajo, liderado por orientadores, } \\
\text { lo que permite brindar una respuesta a la necesidad } \\
\text { de la participación de los encargados legales al } \\
\text { proceso educativo. }\end{array}\end{array}$ & $\begin{array}{l}\text { Los directores delegan la responsabilidad en los } \\
\text { equipos de apoyo. }\end{array}$ \\
\hline
\end{tabular}

En el caso de los talleres, éstos se definieron de acuerdo con las necesidades manifestadas por los participantes, los que determinaron, en cada centro, cuáles eran las 
necesidades de capacitación, según los roles y los perfiles de los docentes. Se definieron, así, los temas más comunes en esta etapa. La Tabla № 7 muestra los aciertos y los desaciertos durante los talleres, y, en el caso de estos últimos, se nota que la hora de inicio de las sesiones no pudo cumplirse, lo que evidencia un problema de programación, tanto por parte de los encargados del proyecto como de los participantes.

En esta etapa, se incluyó el uso del aula virtual que tiene la UNA, con el fin de introducir a los participantes en la práctica de las nuevas Tecnologías de Comunicación (TICS) y, además, poder brindar una opción más para la actualización profesional.

Tabla No. 7

Talleres de actualización para equipos de apoyo y directores

\begin{tabular}{|l|l|}
\hline \multicolumn{1}{|c|}{ Aciertos } & \multicolumn{1}{c|}{ Desaciertos } \\
\hline Temas definidos por los participantes. & $\begin{array}{l}\text { La participación de los directores en las sesiones de } \\
\text { actualización, en ocasiones, se ve afectada por las diversas } \\
\text { convocatorias que reciben de sus jefes inmediatos o } \\
\text { superiores. }\end{array}$ \\
\hline Inclusión del uso del aula virtual. & $\begin{array}{l}\text { Algunos centros educativos carecen de equipo disponible, } \\
\text { para que los docentes puedan conectarse a la red. }\end{array}$ \\
\hline
\end{tabular}

La participación de los miembros de los equipos de apoyo, permitió establecer los enlaces directos con el resto del personal de cada centro. Esto favoreció la comunicación permanente, para la implementación del modelo de gestión apropiado.

\section{Principales hallazgos}

Entre los principales hallazgos de la experiencia, se pueden citar:

- $\quad$ El papel del Director como líder del centro educativo fue indispensable. La participación de los funcionarios de aquellas instituciones que no tuvieron el apoyo de la dirección, 
durante el proceso, se tornó compleja y, en la mayoría de los casos, desertaron. Esta situación nos confirma, una vez más, la importancia de la formación profesional de los directores, en vista de que un centro educativo exitoso, requiere de un liderazgo visionario y con un alto sentido de pertenencia.

- $\quad$ El estilo de liderazgo que demandan los centros educativos debe favorecer ambientes democráticos, en los que los actores sociales generen el sentido de pertenencia.

- Se siente la necesidad de un liderazgo en cuatro dimensiones: estratégico, comunitario, pedagógico y político. Éstos coinciden con el rol que el líder desarrolle, lo que le permite consolidar un verdadero sistema de relaciones. Este sistema le posibilita mantener un cierto equilibrio interno, regular las actividades y los esfuerzos individuales, promover sistemas de participación e implicación de los docentes, crear equipos de trabajo en torno a tareas específicas, generar canales de comunicación, establecer los sistemas de monitoreo, contar con un apoyo para poder administrar los conflictos (Jabif, en Braslavky, Acosta. y Jabif, 2004).

- Los contextos educativos costarricenses están inmersos en una problemática social y política, que está sembrando un sentimiento de desesperanza en muchos educadores que, diariamente, se enfrentan a contextos desafiantes y, muchas veces, amenazantes.

- La gestión en procesos de mejoramiento debe tener su génesis en los centros educativos, de manera ascendente y no descendente, como se ha venido desarrollando, normal e históricamente, en nuestro sistema educativo.

- $\quad$ El trabajo en equipo es esencial en la implementación de innovaciones en los centros educativos. Las experiencias con mayor alcance de indicadores programados, se ubican en instituciones en las que se logra la consolidación de un comité de apoyo identificado y comprometido.

- Los docentes requieren de espacios que les permitan reflexionar acerca de su quehacer, para facilitar la toma de decisiones en la búsqueda de nuevas estrategias y alternativas.

- Clarificar el rol institucional es primordial para la consecución del currículo escolar, ya que éste marca el norte del quehacer en los procesos de aprendizaje y permite su institucionalización. 
- La cultura organizacional de los centros educativos tiene arraigadas algunas creencias y manifestaciones, en torno a la permanencia del "status quo", lo que significa una resistencia negativa hacia los cambios propuestos en torno a los procesos de mejoramiento.

- La programación de centros educativos debe contemplar medianos y largos plazos, pues los resultados de los procesos, por lo general, no son inmediatos. Es por ello que el proceso para la definición y elaboración de un MGP, planteado en esta experiencia resulta efectivo y se puede proyectar a cinco años plazo, aproximadamente.

\section{Recomendaciones para instituciones que deseen desarrollar MGP}

En el desarrollo de procesos educativos no se pueden dar recetas, razón por la cual se dieron las etapas, los aciertos, los desaciertos y los hallazgos, con el fin de que los lectores puedan esquematizar, de manera global, la experiencia desarrollada. Sin embargo, con el único fin de contribuir con el mejoramiento de la calidad de los procesos educativos, se sugieren algunas recomendaciones básicas para iniciar experiencias innovadoras en los centros educativos:

a. El liderazgo del director es indispensable en el desarrollo de MGP. Él es uno de los primeros miembros de la organización que debe tener claro el proceso, para poder facilitar los espacios de reflexión que se requieren.

b. La conformación de un equipo de apoyo es un punto clave para el diseño del MGP; este equipo debe estar conformado por representantes de todos los niveles y las especialidades del centro. Como característica relevante, deben tener un alto nivel de compromiso para con la Institución. Es importante que se nombre un coordinador para el equipo y se brinden los espacios de discusión permanentes, ya que ello facilitará el crecimiento y la madurez de los miembros del equipo. Asimismo, se recomienda utilizar 
un registro de acuerdos para cada una de las sesiones, en el que se registren los asuntos tratados, acuerdos, responsables y tiempos requeridos para su cumplimiento.

c. La etapa diagnóstica es de vital relevancia para la pertinencia del MGP. Los instrumentos diagnósticos pueden ser tan diversos como se deseen; sin embargo, se debe tener claro que dentro de cualquier organización el sistema de información tiene un papel muy importante, en especial, porque para poder llevar a cabo cualquier mejoramiento, se necesitan datos pertinentes. Esta información puede ser histórica, presente o futura (proyecciones o situaciones a las que la organización desea llegar). En el caso del MGP que se busca diseñar, cualesquiera de los subsistemas (diagnóstico, plan estratégico y aseguramiento de calidad) generan y requieren de información precisa y concisa, para el adecuado funcionamiento de cada uno de ellos y del modelo en forma integral.

d. En relación con el proyecto, la existencia de un sistema de información adecuado facilitará la obtención de los datos, dado que permitirá a los miembros de la institución tener la realimentación necesaria para el mejoramiento de cada una de las etapas del proceso que realiza. Esto se debe a que el objetivo final de un sistema de información es el obtener una respuesta positiva, que permita una toma de decisiones eficaz y eficiente.

e. Los resultados de esta etapa diagnóstica se deben compartir con los actores sociales que la suministraron, para hacerlos partícipes en el proceso y que sus ideas, sentimientos y percepciones queden plasmadas en las alternativas curriculares que luego se pretenden desarrollar. Conviene, en esta etapa, buscar estrategias sencillas, que no requieran de recursos tecnológicos complejos, sino que, más bien, lo que se pretende es fomentar la participación y la concreción de ideas, con respecto a la realidad circundante. Otra de las sugerencias, en esta etapa, es agrupar a los actores sociales por intereses, niveles o características: gobiernos estudiantiles, padres de familia, docentes de I Ciclo, docentes de áreas técnicas, entre otros.

f. La elaboración de la misión, la visión y el rol institucional, son indispensables en el proceso, así como también lo es la construcción social de todos los actores participantes, y no la idea, únicamente, del director o equipo de apoyo. 
g. La siguiente etapa es el diseño del MGP, responsabilidad que recae en el equipo de apoyo. Es importante aclarar cuál es la información básica que requieren o desean plasmar en el documento escrito; la introducción, la reseña histórica, el diagnóstico institucional, la misión, la visión, el rol institucional, el marco teórico, los proyectos, las etapas, los recursos, entre otros (Castro, Cerdas, Marín y Vargas, 2007). Es importante mencionar que en esta etapa, se recomienda la utilización de un plan estratégico, el cual tiene como objetivo principal determinar y clarificar hacia dónde quiere dirigirse la organización, y definir, paso a paso, las diferentes etapas o estrategias que deben realizarse para la adecuada administración de los centros educativos.

Finalmente, para que la implementación del MGP sea exitosa, se requiere una comunicación asertiva, un liderazgo basado en principios, toma de decisiones oportunas, trabajo en equipo, creatividad, compromiso y un alto sentido de pertenencia.

\section{Referencias}

Braslavky, C., Acosta, F. y Jabif, L. (2004). Módulos de formación en competencias para la gestión en contextos de pobreza. Orientaciones conceptuales y didácticas y liderazgo. Buenos Aires: IIPE-UNESCO.

Castro, C., Cerdas, V., Marín, A., y Vargas, I. (2007) Desarrollo de Modelos de Gestión Pedagógicos. (Informe parcial 2007) Heredia, Costa Rica: Universidad Nacional: División de Educación para el Trabajo.

Domínguez, G. y Mesanza, J. (1996). Manual de organizaciones educativas. Madrid: Editorial Escuela Española.

Programa de las Naciones Unidas para el Desarrollo, PNUD. (1999). Los retos educativos del futuro. Estado de la Educación de América Latina y el Caribe. San José, Costa Rica: Guilá Imprenta Litografía.

Proyecto Estado de la Nación en Desarrollo Humano Sostenible. (2004). Estado de la Nación en Desarrollo Humano Sostenible. Undécimo informe. San José, Costa Rica: Proyecto Estado de la Nación. 
Proyecto Estado de la Nación en Desarrollo Humano Sostenible. (2005). Estado de la Nación en Desarrollo Humano Sostenible. Duodécimo informe. San José, Costa Rica: Proyecto Estado de la Nación.

\section{Otras fuentes consultadas}

Aguilar, A., Cerdas, V., Marín, A. y Vargas, I. (2006). Actualización académica para administradores educativos en zonas de atención prioritaria. Informe Final 2005-2006. Heredia, Costa Rica: Universidad Nacional.

Chen, E. y Vargas, I. (2007). Administración de recursos institucionales en centros educativos. Heredia, Costa Rica: EUNA.

Dobles, M. C. (1996). Investigación en educación: procesos, interacciones y construcciones. San José, Costa Rica: EUNED.

González, M. T. (2003). Organización y gestión de centros escolares; dimensiones y procesos. Madrid: Pearson.

González, M., Venegas, P. y Madrigal, R. (2006). Proyecto Hemisferio: desarrollo de competencias en la gestión educativa. San José, Costa Rica: Organización de Estados Americanos, Sub región-centroamericana.

Lepeley, M. T. (2001). Gestión y calidad en educación; un modelo de evaluación. Santiago: McGraw-Hill.

Torres, J. (1996). Globalización e interdisciplinariedad: el currículo integrado. Madrid: Morata. 
